Z. Phys. B - Condensed Matter 67, 357-361 (1987)

Condensed

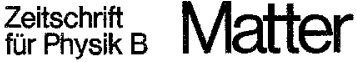

(C) Springer-Verlag 1987

\title{
Wavevector Scaling, Surface Critical Behavior, and Wetting in the 2-d, 3-State Chiral Clock Model
}

\author{
A.L. Stella \\ Dipartimento di Fisica dell' Università and GNSM-CISM, Padova, Italy \\ X.-C. Xie, T.L. Einstein, and N.C. Bartelt \\ Department of Physics and Astronomy, University of Maryland, College Park, Maryland, \\ USA
}

Received December 22, 1986; revised version March 5, 1987

The controversial 2- $d, 3$-state chiral Potts model is studied using transfer matrix finite size scaling. At $\Delta=0$, we find $\mathrm{d} q_{N} / \mathrm{d} \Delta \propto N^{-4 / 5}$, where $q$ is the "wavevector," $\Delta$ the chiral field, and $N$ the strip width $(N=4-10)$. The result is consistent with den Nijs's crossover exponent $\phi=1 / 6$. With surface fields on the infinite free boundaries, exponents associated with bulk magnetization $y_{H}$, surface magnetization $y_{H}^{\prime}$, and surface susceptibility $\chi$ are computed vs. $\Delta$; results are similar for $\hat{\Delta} \|$ or $\perp$ to the infinite direction. Preliminary results are given for the bulk specific heat critical amplitudes, to test the universality of amplitude ratios. The interface wetting line is located for $0 \leqq \Delta \leqq 1 / 4$ using simple transfer matrix calculations of surface tensions in the solid-on-solid approximation. Overhangs or bubbles seem relatively unimportant at all temperatures.

\section{Introduction}

We have undertaken the study of several properties of the chiral 3-state Potts model [1-12]. In spite of the many reports on this model, a full understanding has still eluded statistical mechanicians. Much of this work was motivated by a desire to understand better the melting of a $(3 \times 1)$ overlayer on a centered rectangular substrate, which serves as a prototype of a surface phase with commensurate order which can melt continuously to an incommensurate-disorder phase [13]. Explicitly, the Hamiltonian we consider is [1, 2]

$H=-\sum_{\langle i j\rangle} \cos \left(\frac{2 \pi}{3}\left(n_{i}-n_{j}+\hat{\Delta} \cdot \widehat{R}_{i j}\right)\right), \quad n_{i}=0,1,2$

Because of the symmetries of the free energy, it is well-known that only the regime $0 \leqq \Delta \leqq 1 / 2$ need be treated. There is considerable controversy over whether there is a Lifshitz point [14], as suggested by most numerical studies $[4,6,10,12]$, or whether the incommensurate phase extends down to zero chirality $\Delta[5,9,15]$. Our goals in this work are the following: 1) We determine using transfer matrix finite size scaling [16] how the incommensurability scales as a function of strip width $N$. The motivation is to investigate whether chirality, no matter how small, introduces a new universality class, as suggested by Huse and Fisher [8]. 2) Since chirality changes interface properties dramatically and since surface (i.e. edge) exponents are presumably more sensitive to interfaces than bulk exponents, we compute them, using transfer matrix finite size scaling [17], as a function of 4.3 ). We report on preliminary studies of critical amplitude ratios for the free energy, the first attempt to obtain them within the framework of phenomenological renormalization for a model other than 2- $d$ Ising. Our results for this more elusive property are poorly convergent, but at least as good as Monte Carlo calculations. 4) We calculate the wetting line by computing the surface tension using the solid-on-solid, Müller-Hartmann Zittartz [18] 
(MHZ) approximation, which ignores bubbles and overhangs. We find excellent agreement with Monte Carlo results. We have also obtained some Monte Carlo results for the $(3 \times 1)$ overlayer of central interest, which will be reported elsewhere [13].

\section{Scaling of the Incommensurability for Small Chirality}

As one approaches the critical line from within the incommensurate fluid phase, it is expected that the product of incommensurability $q$ and correlation length $\xi$ approach a finite value, as opposed to being zero within a commensurate phase and infinite in a floating phase. Since according to finite size scaling $\xi \sim N$ at a critical point, one then concludes $q \sim N^{-1}$. Indeed, Duxbury et al. [10] have reported such behavior in transfer matrix scaling. At $\Delta=0, q=0$, even in the disordered regime. The chiral field has been argued to be relevant by a variety of approaches; den Nijs [19] showed that the associated crossover exponent $\phi$ is $1 / 6$. We find using transfer matrix scaling with chirality in either direction (parallel or perpendicular to the infinite direction) that the slow crossover is reflected in notable behavior for the incommensurability at small chirality: $\frac{\mathrm{d} q}{\mathrm{~d} A} \sim N^{-4 / 5}$. Using scaling arguments we show that this exponent is a direct consequence of $\phi=1 / 6$. This is the first numerical illustration, to our knowledge, of this small crossover exponent. We compute the estimator

$\ln \left(\frac{\mathrm{d} q_{N+1}}{\mathrm{~d} A} / \frac{\mathrm{d} q_{N}}{\mathrm{~d} A}\right) / \ln (N / N+1)$.

Using sparse matrices [20], we can obtain an $N$ up through 10 , whereas with symmetry reduction [21] we would be limited to a strip width of 5 or so. Our results are listed in Table 1. Extrapolating our results by fitting 3 consecutive strips to the form $q^{\prime}(N)$ $=q^{\prime}(\infty)+a N^{-x}[15,19,20]$, we obtain 0.7994 for $8-9-10$ and 0.8000 for $7-8-9$, and so $7.997 \pm 0.0003$ for our estimator, indicating $\mathrm{d} q_{N} / \mathrm{d} \Delta \propto N^{-4 / 5}$.

To understand this result, we note [8]

$\xi^{-1} \sim \xi_{r}^{-1}-\mathrm{i} q$,

where $\xi_{\mathrm{r}}^{-1}$ denotes the real part, $\ln \lambda_{0} /\left|\lambda_{1}\right|$.

If we write the crossover form for the correlation length [8]

$\xi=t^{-v} X_{ \pm}\left(\Delta / t^{\phi}\right)$

where $t \sim \frac{T-T_{c}}{T_{c}}$ is the deviation from the critical temperature, we expect [22]
Table 1. Derivative of incommensurability with respect to chirality at zero chirality, for various strip widths

\begin{tabular}{rll}
\hline$N$ & $\mathrm{~d} q / \mathrm{d} \Delta$ & Estimator [cf. (2)] \\
\hline 4 & 0.409908330 & 0.863 \\
5 & 0.338080367 & 0.840 \\
6 & 0.290085517 & 0.826 \\
7 & 0.255423653 & 0.817 \\
8 & 0.229017548 & 0.812 \\
9 & 0.208124756 & 0.809 \\
10 & 0.19112206 & \\
\hline
\end{tabular}

$q \sim t^{v} Y_{ \pm}\left(\Delta / t^{\phi}\right), \quad Y_{ \pm}(0)=0$.

Then

$\left.\frac{\mathrm{d} q}{\mathrm{~d} \Delta}\right|_{\Delta=0} \sim t^{\nu-\phi} \quad$ or $\left.\quad \frac{\mathrm{d} q_{N}}{\mathrm{~d} \Delta}\right|_{T_{c}} \sim N^{-(v-\phi) / v}$

using finite size scaling. Since $v=5 / 6$ for the 3 -state Potts model, $\phi=1 / 6$ implies that the exponent is $-4 /$ 5 , as we found.

It is perhaps worthwhile remarking that the above result for the finite size scaling behavior of $\frac{\mathrm{d} q}{\mathrm{~d} \Delta}$ at $\Delta=0$ does not contradict our expectation that for a finite system at criticality $q \sim N^{-1} \sim \xi^{-1}$ as $N \rightarrow \infty$. Indeed, for such a system, we can always put $q \sim \Delta \frac{\partial q}{\partial \Delta}$ (since $q(A=0)=0$ ), for small $\Delta$. Taking into account that for small $\Delta$ and $t$, and large $N$, the homogeneity of $q$ is:

$q(t, \Delta, N)=l^{-1} q\left(t l^{y_{T}}, \Delta l^{x}, \frac{N}{l}\right)$

with $x=1 / 5$ and $y_{T}=6 / 5$ (characteristic of the 3 -state Potts model), it is easy to realize that, for $t=0, l \sim N$, and $\Delta$ approaching zero as $N^{-1 / 5}$, the last equation implies $\Delta \frac{\partial q}{\partial \Delta} \sim N^{-1}$, as expected.

For the system sizes studied by Duxbury et al., $\bar{\beta}$ (where $q \sim t^{\bar{\beta}}$ ) was poorly convergent for general $\Delta$. Because of the type of crossover behavior discussed above, one expects for each $\Delta$ that a range of $N$ will give poorly convergent estimates of $\bar{\beta}$; only for sufficiently large $N$ does one get beyond the crossover regime. Thus it is conceivable that $\bar{\beta} \approx v$ in the studied system might be a relic of the system crossing over from $\bar{\beta}=\frac{4}{5} v$. The discussion highlights the unusual caution needed in this system in obtaining exponent estimates from a limited range of strip widths. 


\section{Surface Magnetic Exponent}

We expect [17] the singular part of the [dimensionless] free energy per spin of an infinite strip of width $N$ to have the following scaling form:

$f\left(t, h, h_{s}, N\right)=l^{-d} f\left(l^{y_{T}} t, l^{y_{H}} h, l^{y_{H}^{\prime}} h_{s}, N / l\right)$

where $h_{s}$ is field applied only at the surfaces (edges) of the strip and $y_{H}^{\prime}$ is the associated exponent. Then, at criticality, using standard finite size scaling arguments [17], we have

$$
\begin{aligned}
& \chi \equiv \frac{\partial^{2} f}{\partial h^{2}} \sim N^{-d+2 y_{H X}} \\
& \frac{\partial^{2} f}{\partial h \partial h_{s}} \sim N^{-d+y_{H}+y_{H}^{\prime}} \equiv N^{x} .
\end{aligned}
$$

For finite chirality, $T_{c}$ was estimated by invoking the phenomenological matching condition for the bulk susceptibility $\chi$ using periodic boundary conditions: We computed $[10,23]$ the $X^{N}(T)$ $\equiv \ln \left(\chi^{N+1} / \chi^{N}\right) / \ln ((N+1) / N)$ and determined $T_{c}$ from the condition $X^{N}\left(T_{c}\right)=X^{N+1}\left(T_{c}\right)$ for $N=5$, i.e. strips of width 5,6 , and 7 . This approach proved better convergent than estimates based on the more typical scaling condition for the correlation length, and circumvented the issue of possible anisotropic scaling $[13,24]$.

We next computed at this $T_{c}$, with free boundary conditions, $y_{H}, x$, and thence $y_{H}^{\prime}$, as listed in Table 2 . Not unexpectedly, convergence is poorer than with periodic boundary conditions, even for the bulk expo-

Table 2. Magnetic exponents as functions of chirality $\Delta$, both parallel and perpendicular to the infinite direction. In the parallel case, the listed values of $y_{H}$ and $x$ are from the largest pair of strips used $(6,7)$. Then $Y_{\tilde{H}}=d+x-y_{H}$ was computed for this pair and the two next smaller pairs, $(5,6)$ and $(4,5)$; the tabulated value is from a power-law extrapolation. For the perpendicular case, $y_{H}$ is the value extrapolated from $(3,4),(4,5)$, and $(5,6)$

\begin{tabular}{lllll}
\hline & $\Delta$ & $y_{H}$ & $x$ & $y_{H}^{\prime}$ \\
\hline Exact & 0 & 1.867 & 0.2 & $1 / 3$ \\
Paral. & 0 & 1.82 & 0.198 & 0.366 \\
& 0.05 & 1.82 & 0.196 & 0.367 \\
& 0.10 & 1.82 & 0.208 & 0.382 \\
& 0.15 & 1.83 & 0.233 & 0.403 \\
& 0.20 & 1.83 & 0.252 & 0.422 \\
Perp. & 0.05 & 1.83 & 0.209 & 0.377 \\
& 0.10 & 1.83 & 0.208 & 0.376 \\
& 0.15 & 1.83 & 0.210 & 0.379 \\
& 0.20 & 1.83 & 0.209 & 0.383 \\
& 0.25 & 1.82 & 0.204 & 0.388 \\
& 0.30 & 1.79 & 0.192 & 0.398 \\
\hline
\end{tabular}

nent $y_{H}$. (One generally expects that the leading finitesize correction in $f$ for periodic boundary conditions is $\mathscr{O}\left(N^{-2}\right)$ while for free boundaries there is a surface term $\left.\mathcal{O}\left(N^{-1}\right).\right)^{25}$ In the case of zero chirality we obtained reasonable agreement with known exponents [17]. For finite $\Delta$, we did calculations with chirality both parallel to and perpendicular to the infinite direction. For a given relatively modest numerical effort, we could go to larger widths in the former case, as shown in the table. While in general TMS studies of the effects of chirality, one expects better results when this field is in the infinite direction, it is not clear that this superiority still holds when one is interested in surface properties. Consistent with previous numerical studies, nothing dramatic happens for small $\Delta$, while for larger $\Delta$ convergence breaks down. This behavior is consistent with both 1) no change in exponents due to the introduction of weak chirality and 2) immediate very slow crossover consistent with den Nijs's result $\phi=1 / 6$. It is noteworthy that there is little difference between the two directions of chirality, when $A$ is small.

\section{Free Energy Critical Amplitude Ratio}

We mention some preliminary calculations of free energy critical amplitudes generalizing a procedure first outlined by Nightingale [26]. For an infinite system we expect [27]

$f(t)=g(t)+l^{-d} f\left(t^{(1)}(t)\right)$

where $g(t)$ is regular and $t^{(1)}$ is the first iterate of $t$ under renormalization. The idea is to obtain $g$ from transfer matrix finite size scaling. Even if $g$ is known only approximately, the finite summation and the presence of an unstable fixed point lead to nontrivial approximate $f$ 's. For a strip of width $N$, then, we approximate

$g(t) \simeq f_{N}(t)-l^{-d} f_{N / l}\left(t^{(1)}(t)\right)=g_{0}+g_{1} t+\ldots$

with $t^{(1)}(t)$ given implicitly by the phenomenological scaling equation

$\xi_{N}(t)=l \xi_{N / l}\left(t^{(1)}(t)\right)$.

Writing $f$ as a sum of a regular and singular part, $f^{r}$ and $f^{\text {sing }}$ respectively, with $f^{r}=f_{0}+f_{1} t+\ldots$ and $f=f^{r}+f^{\text {sing }}$, with $f^{\text {sing }} \simeq A_{ \pm}|t|^{d / y_{T}}$, it is straightforward to show

$A \simeq\left(f(t)-f_{0}^{r}-f_{1}^{r} t\right)|t|^{-d / y_{T}}$

with 
$f_{0}^{r}=\frac{g_{0}}{1-l^{-d}}, \quad f_{1}^{r}=\frac{g_{1}}{1-l^{-d+y_{T}}}, \quad$ and

$f(t)=\sum_{n=0}^{\infty} l^{-n d} g\left(t^{(n)}(t)\right)$

where $t^{(n)}$ is the $n$-th iterate under the renormalization transformation. Thus, while $g_{0}$ and $g_{1}$ are computed "locally" at the fixed point, $f(t)$ must be computed along the RG trajectory. Applying this scheme to the pure 3-state Potts model yields estimates of the amplitude ratio $A_{+} / A_{-}$of $1.340,1.259$ and 1.194 for strip widths 4,5 , and 6 , respectively. The extrapolated ratio is 0.925 , in comparison with the exact value of unity, known from duality [28]. While the level of accuracy does not indicate great quantitative promise, the agreement is to within $10 \%$, which compares favorably with $20 \%$ or worse error bars for amplitude ratios obtainable in Monte Carlo simulations of lattices of sizes typical of experiments (and thus indicative of what might be measured) [13]. For $\Delta=0.1$ we computed $A_{+} / A_{-}$, finding values of 1.357 and 1.268 for $N=5$ and 6 , respectively. While these numbers are quite inconclusive regarding the presence of a Lifshitz point, they are consistent with the general theme of numerical results that nothing dramatic happens as weak chirality is introduced.

In further justification of these calculations, we note the difficulty of trying to obtain a universal number by computing two non-universal numbers and taking their ratio.

This procedure involves global renormalization flow features, rather than local properties at the fixed point, characteristic of critical exponents. The excellent convergence obtained by Nightingale [26] for the nearest-neighbor Ising model is not surprising: the exceptional occurrence of a logarithmic singularity for this special model avoids the need to estimate the free energy from a sum over the RG trajectory [27] (i.e. the $f(t)$ in (14)); only the first and second derivatives of $g(t)$ and of the transformations $t^{(n)}$ at criticality need be calculated.

\section{Wetting Line}

In this section we compute the surface tension (interface free energy) $\sigma$ using the Müller-Hartmann Zittarz (MHZ) method and use $\sigma$ to determine the wetting line [29]. This approximation allows only solid-onsolid excitations, excluding overhangs along the boundary between different phases and bubbles within phases. Selke and Pesch [30] applied this method to locate the critical line with the condition $\sigma=0$. In this problem, we estimate the wetting line from the Antonow [31] condition $\sigma_{0-2}\left(T_{w}\right)=2 \sigma_{0-1}\left(T_{w}\right)$, used e.g. by Huse and Fisher [8]. A similar approach,

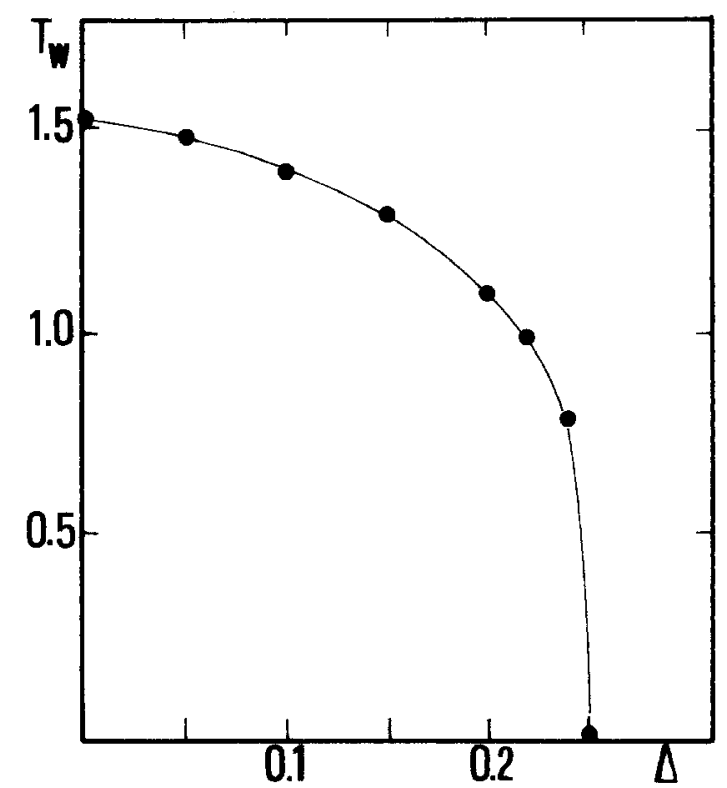

Fig. 1. Values of $T_{W}$ as a function of $\Delta$ determined in the present paper

appearing recently, was taken for the ANNNI model [32]. To compute $\sigma$ between two phases, we follow Selke and Pesch's treatment of the 3-state Potts model, writing down a transfer matrix in terms of the size of an intercalated third phase at the interface and the coordinate at one end of the intercalate. The largest eigenvalue of this transfer matrix then determines $\sigma$ as usual. The difference in the end coordinates between neighboring columns can be summed analytically, leading to their [30] Eq. (4). For the chiral field normal to the interface, only minor modification of a simple prefactor is needed. Indeed, Selke and Pesch generated as an aside a curve of the transition temperature for the chiral clock model and noted its superiority to the free-fermion approximation. To proceed we limited the intercalate length to 49 and evaluated the eigenvalues of the resulting matrix with a packaged program. Our results are sketched in Fig. 1; they agree well with those obtained by Yeomans and Derrida [12] via scaling of the "net adsorption" (i.e. occupation of 1 between 0 and 2 regions). The agreement with Monte Carlo results reported by Selke [33] is also excellent. By construction, our approximation gives the wetting temperature as the SOS critical temperature in the zero-chirality limit. (i.e. $\sigma\left(T_{w}\right)=0$ ); Selke and Pesch [30] noted that this $T_{c}$ of $1.53 \mathrm{com}-$ pares well with the exact result of 1.4925 . Yeomans and Derrida also computed by scaling methods the wetting line for a solid-on-solid model expected to be in the same universality class as the chiral Potts model. Their results are good for low temperature but the wetting curve rises sharply with decreasing $\triangle$, behavior they attribute to the absence of a diverging bulk correlation length. They also find a wetting 
exponent consistent with unity for this model. We believe that this behavior comes from the fact that the SOS approximation allows one to compute the surface free energy (tension) from a one-dimensional model; it is hard to see how non-trivial exponents could emerge from such a model.

\section{Concluding Remarks}

The results of the present investigation leave us still far from the goal of determining correctly and completely the phase diagram and critical behavior of the chiral 3-state Potts model. Nevertheless we believe that we have made clear progress concerning some particular but important issues, which were either ignored or not carefully considered by the previous literature. In the second section we obtained the first numerical confirmation of the slow crossover due to chirality, as predicted by den Nijs.

The determination of surface critical exponents for finite chirality is an interesting test which was never applied before. That these results evidently do not allow clear conclusions, like for the bulk case, is a further indication of the puzzling nature of the model. What seems to be apparent is that chirality does not lead to the suspected strong effects on the scaling properties of the surface free energy.

Some preliminary progress is reported of an attempt to gain quantitative insight about the amplitude ratio of the model by phenomenological scaling methods. A serious motivation for this development was to test possible different universality domains for different chiralities in the model. In M.C. calculations the accuracy one can get for these ratios is not adequate $(\sim 20 \%)[13,34]$. The situation is not really significantly better in a TMS framework; new ideas seem to be needed to improve the computational power.

The calculations in the last section show that at least the wetting aspects of the model can receive a reasonably convincing global description within a single and physically sound approximation. As to the problem of wetting exponents [12], however, we notice that they should be addressed in a more realistic framework, because the SOS approximation cannot lead to nontrivial scaling for the surface tension.

Work at University of Maryland supported by U.S. Department of Energy under Grant \# DE-FG05-84ER 45071. ALS acknowledges the hospitality of the University of Maryland during Fall 1985. TLE acknowledges the hospitality of the University of Padova in July 1986 and support from Unità GNSMCISM and as well as a travel assistance award from the University of Maryland. During the later stages, we received support from NATO Grant No. 86/0782. One of us (TLE) benefitted from several helpful discussions with $J$. Amar and an insightful comment by P. Bak.

\section{References}

1. Ostlund, S.: Phys. Rev. B24, 398 (1981)

2. Huse, D.A.: Phys. Rev. B 24, 5180 (1981)

3. Yeomans, J.M., Fisher, M.E.: J. Phys. C14, L835 (1981)

4. Selke, W., Yeomans, J.M.: Z. Phys. B - Condensed Matter 46, $311(1982)$

5. Kardar, M.: Phys. Rev. B26, 2693 (1982)

6. Howes, S.F.: Phys. Rev. B27, 1762 (1983)

7. Huse, D.A., Szpilka, A., Fisher, M.E.: Physica 121 A, 363 (1983)

8. Huse, D.A., Fisher, M.E.: Phys. Rev. B29, 239 (1984); Phys. Rev. Lett. 49, 7983 (1982)

9. Gehlen, G. von, Rittenberg, V.: Nucl. Phys. B230 [FS 10], 455 (1984) studied the quantum version of the 3-clock model, contrary to many citations of their work, they found a floating phase at no (rather than every non-zero) chirality

10. Duxbury, P.M., Yeomans, J., Beale, P.D.: J. Phys. A 17, L 179 (1984)

11. Yeomans, J.: Physica 127B, 187 (1984)

12. Yeomans, J., Derrida, B.: J. Phys. A 18, 2343 (1985)

13. Barteit, N.C., Einstein, T.L., Roelofs, L.D.: Phys. Rev. B 35, $4812(1987)$

14. Hornreich, M., Luban, M., Shtrikman, S.: Phys. Rev. Lett. 35, $1678(1975)$

15. Haldane, F.D.M., Bak, P., Bohr, T.: Phys. Rev. B 28, 2743 (1983); Schulz, H.J.: Phys. Rev. B28, 2746 (1983)

16. Nightingale, M.P.: J. Appl. Phys. 53, 7927 (1982)

17. Droz, M., Malaspinas, A., Stella, A.L.: J. Phys. A 18, L245 (1985)

18. Müller-Hartmann, E., Zittarz, J.: Z. Phys. B - Condensed Matter and Quanta 27, 261 (1977)

19. Nijs, M. den: J. Phys. A 17, L 295 (1984)

20. Nightingale, M.P.: Proc. K. Ned. Akad. Wet. B82, 235 (1979)

21. Kinzel, W., Schick, M.: Phys. Rev. B23, 3435 (1981); B24, 324 (1981)

22. Huse, D.A.: Private communication 1984

23. Schaub, B., Domany, E.: Phys. Rev. 28, 2897 (1983)

24. Kinzel, W.: Phys. Rev. Lett. 51, 996 (1983)

25. Barber, M.N.: In: Phase transitions and critical phenomena. Domb, C., Lebowitz, J.L. (eds.), Vol. 8, Chap. 2. London: Academic Press 1983

26. Nightingale, M.P.: Physica 83A, 561 (1976)

27. Niemeijer, Th., Leeuwen, J.M.J. van: In: Phase transitions and critical phenomena. Domb, C., Green, M.S. (eds.), Vol. 6. London: Academic Press 1976

28. Kaufman, M., Andelman, D.: Phys. Rev. B29, 4010 (1984)

29. Fisher, M.E.: J. Chem. Soc. Faraday Transact. II 82, 1569 (1986)

30. Selke, W., Pesch, W.: Z. Phys. B - Condensed Matter 47, 335 (1982)

31. Rowlinson, J.S., Widom, B.: Molecular theory of capillarity. Oxford: Clarendon Press 1982 cited by Ref. 29

32. Ala-Nissila, T., Amar, J., Gunton, J.D.: J. Phys. A 19, L41 (1986)

33. Selke, W.: Surf. Sci. 144, 176 (1984); Static critical phenomena in inhomogeneous systems. In: Lecture Notes in Physics. Pekalski, A., Sznaid, J. (eds.), Vol. 206, Berlin, Heidelberg, New York: Springer 1984

34. Bartelt, N.C., Einstein, T.L., Roelofs, L.D.: Phys. Rev. B35, 1776 (1987)

A.L. Stella

Dipartimento di Fisica

dell'Università di Padova

Via F. Marzolo, 8

I-35131 Padova

Italy

X.-C. Xie, T.L. Einstein, N.C. Bartelt

Department of Physics and Astronomy

University of Maryland

College Park, MD 20742

USA 